\title{
Approximate Solutions for Flow with a Stretching Boundary due to Partial Slip
}

\author{
U. Filobello-Nino, ${ }^{1}$ H. Vazquez-Leal, ${ }^{1}$ A. Sarmiento-Reyes, ${ }^{2}$ B. Benhammouda, ${ }^{3}$ \\ V. M. Jimenez-Fernandez, ${ }^{1}$ D. Pereyra-Diaz, ${ }^{1}$ A. Perez-Sesma, ${ }^{1}$ J. Cervantes-Perez, \\ J. Huerta-Chua, ${ }^{4}$ J. Sanchez-Orea, ${ }^{1}$ and A. D. Contreras-Hernandez ${ }^{1}$ \\ ${ }^{1}$ Electronic Instrumentation and Atmospheric Sciences School, University of Veracruz, Circuito Gonzalo Aguirre Beltrán S/N, \\ 91000 Xalapa, VER, Mexico \\ ${ }^{2}$ National Institute for Astrophysics, Optics and Electronics, Luis Enrique Erro No. 1, Santa Maria Tonantzintla, \\ 72840 Puebla, PUE, Mexico \\ ${ }^{3}$ Abu Dhabi Men's College, Higher Colleges of Technology, P.O. Box 25035, Abu Dhabi, UAE \\ ${ }^{4}$ Civil Engineering School, University of Veracruz, Venustiano Carranza S/N, Colonia Revolucion, 93390 Poza Rica, VER, Mexico \\ Correspondence should be addressed to H. Vazquez-Leal; hvazquez@uv.mx
}

Received 1 August 2014; Revised 26 October 2014; Accepted 26 October 2014; Published 24 November 2014

Academic Editor: Weimin Han

Copyright (C) 2014 U. Filobello-Nino et al. This is an open access article distributed under the Creative Commons Attribution License, which permits unrestricted use, distribution, and reproduction in any medium, provided the original work is properly cited.

The homotopy perturbation method (HPM) is coupled with versions of Laplace-Padé and Padé methods to provide an approximate solution to the nonlinear differential equation that describes the behaviour of a flow with a stretching flat boundary due to partial slip. Comparing results between approximate and numerical solutions, we concluded that our results are capable of providing an accurate solution and are extremely efficient.

\section{Introduction}

According to the classification of Prandtl, the fluid motion is divided into two regions. The first region is near the object where the effect of friction is important and is known as the boundary layer, while, for the second type, these effects can be neglected [1-3]. It is common to define the boundary layer as the region where the fluid velocity parallel to the surface is less than $99 \%$ of the free stream velocity [1].

The boundary layer thickness $\delta$ increases from the edge along the surface on which the fluid moves. Even for the case of a laminar flow, the exact solution of equations describing the laminar boundary layer is very difficult to calculate and only few simple problems can straightforward be analysed [1, 3].

An interesting case is the one where a flow is induced into a viscoelastic fluid by a linearly stretched sheet [4-6] (see Figure 1). Extrusion of molten polymers through a slit die for the production of plastic sheets is an important process in polymer industry [4]. The process is normally complicated from the physical point of view, because it requires significant heat transfer between the sheet and a surrounding fluid that plays the role of a cooling medium. An important aspect of the flow is the extensibility of the sheet which can be employed to improve its mechanical properties along the sheet. To obtain better results is necessary to improve the cooling rate, whereby it is common to add some polymeric additives into water (which is one of the most employed fluids as cooling medium) in order to have a better control on the cooling rate. The flow due to a stretching boundary is also important in other engineering processes of interest, such as the glass fibre drawing and crystal growing among many others. Detail discussion about this topic can be found in [4]. This work assumes that the boundary conditions for the problem under study are adequately described by Navier's condition, which states that the amount of relative slip is proportional to local shear stress. Unlike what happens with fluids like water, mercury, and glycerine, which do not require 
slip boundary conditions [7], there are cases where partial slip between the fluid and the moving surface may occur. Some known cases include emulsions, as mustard and paints and polymer solutions and clay [7].

He $[8,9]$ proposed the standard HPM; it was introduced as a powerful tool to approach several kinds of nonlinear problems. The HPM can be considered as a combination between the classical perturbation technique and the homotopy (whose origin is in the topology), but not restricted to the limitations found in traditional perturbation methods. For instance, HPM method does need neither small parameter nor linearisation, just few iterations to obtain accurate results [5, 8-35]. The fundamentals of HPM convergence can be found in $[21,24,25]$.

There are other modern alternatives to find approximate solutions to the differential equations that describe some nonlinear problems such as those based on variational approaches [36-39], tanh method [40], exp-function [41, 42], Adomian decomposition method [43-49], parameter expansion [50], homotopy analysis method [4, 51, 52], and perturbation method [53] among many others.

To figure out how HPM method works, consider a general nonlinear equation in the form

$$
A(u)-f(r)=0, \quad r \in \Omega,
$$

with the following boundary conditions:

$$
B\left(u, \frac{\partial u}{\partial n}\right)=0, \quad r \in \Gamma,
$$

where $A$ is a general differential operator, $B$ is a boundary operator, $f(r)$ is a known analytical function, and $\Gamma$ is the domain boundary for $\Omega$.

Also, $A$ can be divided into two parts $L$ and $N$, where $L$ is linear and $N$ nonlinear; from this last statement, (1) can be rewritten as

$$
L(u)+N(u)-f(r)=0 .
$$

In a broad sense, a homotopy can be constructed in the following form $[8,9]$ :

$$
\begin{aligned}
H(v, p)= & (1-p)\left[L(v)-L\left(u_{0}\right)\right] \\
+p[L(v)+N(v)-f(r)]=0, & \\
& p \in[0,1], \quad r \in \Omega,
\end{aligned}
$$

or

$$
\begin{array}{r}
H(v, p)=L(v)-L\left(u_{0}\right)+p\left[L\left(u_{0}\right)+N(v)-f(r)\right]=0, \\
p \in[0,1], \quad r \in \Omega,
\end{array}
$$

where $p$ is a homotopy parameter, whose values are within range of 0 and 1 , and $u_{0}$ is the first approximation to the solution of (3) that satisfies the boundary conditions. Assuming that solution for (4) or (5) can be written as a power series of $p$

$$
v=v_{0}+v_{1} p_{1}+v_{2} p_{2}^{2}+\cdots
$$

Substituting (6) into (5) and equating identical powers for $p$ terms, it is possible to obtain the values for the sequence $u_{0}, u_{1}, u_{2}, \ldots$

When $p \rightarrow 1$, it yields in the approximate solution for (1) in the form

$$
v=v_{0}+v_{1}+v_{2}+v_{3}+\cdots .
$$

Another way to build a homotopy, which is relevant for this paper, is by considering the following general equation:

$$
L(v)+N(v)=0
$$

where $L(v)$ and $N(v)$ are the linear and nonlinear operators, respectively. It is desired that solution for $L(v)=0$ describes, accurately, the original nonlinear system.

By the homotopy technique, a homotopy is constructed as follows [18]:

$$
(1-p) L(v)+p[L(v)+N(v)]=0 .
$$

Again, it is assumed that solution for (9) can be written in the form (6); thus, taking the limit when $p \rightarrow 1$ results in the approximate solution for (8).

The variation of homotopic parameter within the range $[0,1]$ amounts to a deformation that begins from an initial equation with known solution until it becomes the equation to be solved. From a practical point of view, taking the limit $p \rightarrow 1$ is just setting $p=1$.

\section{Padé Approximant}

Let $u(t)$ be an analytical function with the Maclaurin's expansion

$$
u(t)=\sum_{n=0}^{\infty} u_{n} t^{n}, \quad 0 \leq t \leq T
$$

Then the Padé approximant to $u(t)$ of order $[L, M]$ which we denote by $[L / M]_{u}(t)$ is defined by [54-57]

$$
\left[\frac{L}{M}\right]_{u}(t)=\frac{p_{0}+p_{1} t+\cdots+p_{L} t^{L}}{1+q_{1} t+\cdots+q_{M} t^{M}},
$$

where we considered $q_{0}=1$, and the numerator and denominator have no common factors.

The numerator and the denominator in (11) are constructed so that $u(t)$ and $[L / M]_{\mathcal{u}}(t)$ and their derivatives agree at $t=0$ up to $L+M$. That is,

$$
u(t)-\left[\frac{L}{M}\right]_{u}(t)=O\left(t^{L+M+1}\right) .
$$

From (12), we have

$$
u(t) \sum_{n=0}^{M} q_{n} t^{n}-\sum_{n=0}^{L} p_{n} t^{n}=O\left(t^{L+M+1}\right) .
$$


From (13), we get the following algebraic linear systems:

$$
\begin{aligned}
& u_{L} q_{1}+\cdots+u_{L-M+1} q_{M}=-u_{L+1} \\
& u_{L+1} q_{1}+\cdots+u_{L-M+2} q_{M}=-u_{L+2} \\
& \vdots \\
& u_{L+M-1} q_{1}+\cdots+u_{L} q_{M}=-u_{L+M}, \\
& p_{0}=u_{0} \\
& p_{1}=u_{1}+u_{0} q_{1} \\
& \vdots \\
& p_{L}=u_{L}+u_{L-1} q_{1}+\cdots+u_{0} q_{L} .
\end{aligned}
$$

From (14), we calculate first all the coefficients $q_{n}, 1 \leq n \leq M$. Then, we determine the coefficients $p_{n}, 0 \leq n \leq L$ from (15).

Note that for a fixed value of $L+M+1$, the error (12) is the smallest when the numerator and denominator of (11) have the same degree or when the numerator has one degree higher than the denominator.

\section{Laplace-Padé Resummation Method}

Several approximate methods provide power series solutions (polynomial). Nevertheless, sometimes, this type of solutions lacks large domains of convergence. Therefore, Laplace-Padé resummation method [54] is used in literature to enlarge the domain of convergence of solutions or inclusive to find exact solutions.

The Laplace-Padé method can be explained as follows.

(1) First, Laplace transform is applied to power series.

(2) Next, $s$ is substituted by $1 / t$ in the resulting equation.

(3) After that, we convert the transformed series into a meromorphic function by forming its Padé approximant of order $[N / M] . N$ and $M$ are arbitrarily chosen, but they should be of smaller values than the order of the power series. In this step, the Padé approximant extends the domain of the truncated series solution to obtain better accuracy and convergence.

(4) Then, $t$ is substituted by $1 / s$.

(5) Finally, by using the inverse Laplace $s$ transform, we obtain the exact or approximate solution.

\section{Formulation}

Consider a two-dimensional stretching boundary (see Figure 1). Experiments show that the velocity of the boundary $U$ is approximately proportional to the distance from the orifice $X$ $[7,58]$, so that

$$
U=b X
$$

where $b$ is a proportionality constant.

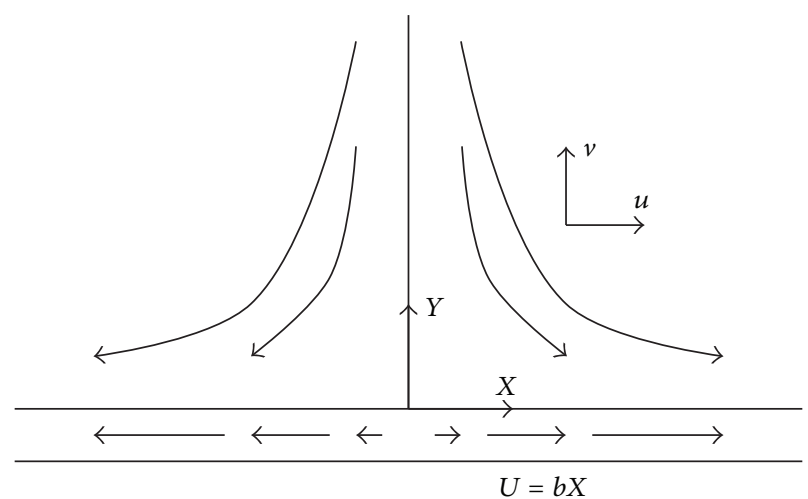

FIGURE 1: Schematic showing a stretching boundary.

Let $(u, v)$ be the fluid velocities for the $(X, Y)$ directions, respectively. In this case the boundary condition is adequately described by Navier's condition, which states that the amount of relative slip is proportional to local shear stress:

$$
u(X, 0)-U=k \nu \frac{\partial u}{\partial Y}(X, 0)
$$

where $k$ is a constant of proportionality and $v$ is the kinematic viscosity of the bulk fluid. The relevant expressions for this case are the Navier-Stokes equations:

$$
\begin{gathered}
u u_{X}+v u_{Y}+\frac{p_{X}}{\rho}-v\left(u_{X X}+u_{Y Y}\right)=0 \\
u v_{X}+v v_{Y}+\frac{p_{Y}}{\rho}-v\left(v_{X X}+v_{Y Y}\right)=0
\end{gathered}
$$

and continuity

$$
u_{X}+v_{Y}=0
$$

where $\rho$ and $p$ are density and pressure, respectively.

To solve equations (18)-(20), we have to consider boundary conditions (16) and (17), besides the fact that there is no lateral velocity or pressure gradient away from the stretching surface.

Next, we will show that it is possible to get an ordinary nonlinear differential equation from (18).

With this end, we note that $Y$ component of velocity is negative $(v<0)$ and by symmetry arguments it only depends on $Y$ (see Figure 1). Therefore, it is possible to define a function $y(x) \geq 0,0 \leq x<\infty$, such that [7]:

$$
v=-\sqrt{b v} y(x)
$$

where

$$
x=Y \sqrt{\frac{a}{v}} .
$$

From (21) and continuity equation (20) we obtain

$$
u=b X y^{\prime}(x) \text {. }
$$


Clearly (20) is satisfied under transformations (21), (22), and (23), while (18) adopts the simpler form;

$$
y^{\prime \prime \prime}(x)-\left(y^{\prime}(x)\right)^{2}+y(x) y^{\prime \prime}(x)=0
$$

To deduce the boundary conditions of (24), we see from Figure 1 that $v(Y=0)=0$ and $\lim _{Y \rightarrow \infty} u(X, Y)=0$ and therefore

$$
\begin{array}{r}
y(0)=0, \\
y^{\prime}(\infty)=0 .
\end{array}
$$

Finally, substituting (16) and (23) into (17) we obtain

$$
y^{\prime}(0)=k \sqrt{b v} y^{\prime \prime}(0)+1
$$

\section{Approximate Solution for a Two-Dimensional Viscous Flow Equation}

Next, we present some solution methods to the study problem.

5.1. HPM Method. In this section, HPM is used to find approximate solutions for (24). Identifying the linear part as

$$
L=y^{\prime \prime \prime}
$$

and the nonlinear as

$$
N=-\left(y^{\prime}\right)^{2}+y y^{\prime \prime}
$$

we initiate the HPM method by constructing a homotopy based on (9), in the form

$$
(1-p) y^{\prime \prime \prime}+p\left(y^{\prime \prime \prime}-\left(y^{\prime}\right)^{2}+y y^{\prime \prime}\right)=0
$$

Assuming that the solution has the form $[8,9]$ :

$$
y=y_{0}+y_{1} p_{1}+y_{2} p_{2}^{2}+\cdots
$$

Then, substituting (30) into (29) and equating terms having identical powers of $p$ we obtain

$$
\begin{aligned}
& p^{0}: y_{0}^{\prime \prime \prime}=0, \\
& p^{1}: y_{1}^{\prime \prime \prime}-\left(y_{0}^{\prime}\right)^{2}+y_{0} y_{0}^{\prime \prime}=0, \\
& p^{2}: y_{1} y_{0}^{\prime \prime}-2 y_{0}^{\prime} y_{1}^{\prime}+y_{2}^{\prime \prime \prime}+y_{0} y_{1}^{\prime \prime}=0, \\
& p^{3}: y_{2}^{\prime \prime}+y_{1} y_{1}^{\prime \prime}-y_{1}^{\prime 2}+y_{2} y_{0}^{\prime \prime}+y_{3}^{\prime \prime \prime}-2 y_{0}^{\prime} y_{2}^{\prime}=0, \\
& p^{4}: y_{1}^{\prime \prime}+y_{0} y_{3}^{\prime \prime}+y_{1} y_{2}^{\prime \prime}-2 y_{0}^{\prime} y_{3}^{\prime}+y_{3} y_{0}^{\prime \prime}+y_{4}^{\prime \prime \prime} \\
& \quad-2 y_{1}^{\prime} y_{2}^{\prime}=0,
\end{aligned}
$$

$$
\begin{aligned}
& p^{5}: y_{3}^{\prime \prime}-y_{2}^{\prime 2}+y_{5}^{\prime \prime \prime}-2 y_{1}^{\prime} y_{3}^{\prime}+y_{4} y_{0}^{\prime \prime}+y_{2} y_{2}^{\prime \prime}-2 y_{0}^{\prime} y_{4}^{\prime} \\
& \quad+y_{0} y_{4}^{\prime \prime}+y_{3} y_{1}^{\prime \prime}=0, \\
& p^{6}: y_{5} y_{0}^{\prime \prime}+y_{0} y_{5}^{\prime \prime}+y_{6}^{\prime \prime \prime}-2 y_{0}^{\prime} y_{5}^{\prime}+y_{4} y_{1}^{\prime \prime}+y_{1} y_{4}^{\prime \prime} \\
& \quad+y_{2} y_{3}^{\prime \prime}-2 y_{1}^{\prime} y_{4}^{\prime}+y_{3} y_{2}^{\prime \prime}-2 y_{2}^{\prime} y_{3}^{\prime}=0, \\
& p^{7}: y_{4}^{\prime \prime}-y_{3}^{\prime 2}+y_{4} y_{2}^{\prime \prime}+y_{1} y_{5}^{\prime \prime}+y_{0} y_{6}^{\prime \prime}+y_{6} y_{0}^{\prime \prime}-2 y_{2}^{\prime} y_{4}^{\prime} \\
& \quad+y_{5} y_{1}^{\prime \prime}+y_{3} y_{3}^{\prime \prime}-2 y_{0}^{\prime} y_{6}^{\prime}+y_{7}^{\prime \prime \prime}-2 y_{1}^{\prime} y_{5}^{\prime}=0 .
\end{aligned}
$$

In order to fulfil the boundary conditions from (24) given by (25)-(26), we find that $y_{0}(0)=0, y_{0}^{\prime}(0)=1+k \sqrt{b v} a$, $y_{0}^{\prime \prime}(0)=a, y_{1}(0)=0, y_{1}^{\prime}(0)=0, y_{1}^{\prime \prime}(0)=0, y_{2}(0)=0, y_{2}^{\prime}(0)=$ $0, y_{2}^{\prime \prime}(0)=0, y_{3}(0)=0, y_{3}^{\prime}(0)=0, y_{3}^{\prime \prime}(0)=0$, and so on. We have assumed the value for $y^{\prime \prime}(0)$ as some adequate constant $a$, which adopts the following values for the corresponding $k$ as follows: $\{k=0, a=-1\},\{k=0.3, a=-0.701\}$, $\{k=1, a=-0.430\},\{k=2, a=-0.284\},\{k=5, a=-0.145\}$, and $\{k=20, a=-0.0438\}$ [6]. Thus, the results obtained from above equations are

$$
\begin{aligned}
& y_{0}=x\left(1+\left(\frac{1}{2} x+k\right) a\right) \\
& y_{1}=\frac{1}{6}\left(1+\left(\frac{1}{4} x k+\frac{1}{20} x^{2}+k^{2}\right) a^{2}+\left(2 k+\frac{1}{4} x\right) a\right) x^{3} \text {, } \\
& y_{2}=\frac{a x^{6}}{720}\left(1+\left(-\frac{1}{7} x k-\frac{1}{56} x^{2}+k^{2}\right) a^{2}\right. \\
& \left.+\left(2 k-\frac{1}{7} x\right) a\right) \\
& y_{3}=\frac{x^{7}}{2520}\left(1+\left(\frac{1}{8} x k^{3}+\frac{3}{160} x^{3} k\right.\right. \\
& \left.+\frac{1}{16} x^{2} k^{2}+\frac{3}{1760} x^{4}+k^{4}\right) a^{4} \\
& +\left(\frac{3}{160} x^{3}+4 k^{3}+\frac{1}{8} x^{2} k+\frac{3}{8} x k^{2}\right) a^{3} \\
& \left.+\left(6 k^{2}+\frac{1}{16} x^{2}+\frac{3}{8} x k\right) a^{2}+\left(4 k+\frac{1}{8} x\right) a\right), \\
& y_{4}=-\frac{x^{9}}{45360}\left(1+\left(\frac{13}{40} x k^{4}+\frac{29}{440} x^{2} k^{3}+\frac{317}{640640} x^{5}\right.\right. \\
& \left.+\frac{101}{3520} x^{3} k^{2}+\frac{317}{45760} x^{4} k+k^{5}\right) a^{5} \\
& +\left(\frac{87}{440} x^{2} k^{2}+5 k^{4}+\frac{13}{10} x k^{3}\right. \\
& \left.+\frac{317}{45760} x^{4}+\frac{101}{1760} x^{3} k\right) a^{4} \\
& \left.+\left(\frac{87}{440} x^{2} k+\frac{39}{20} x k^{2}+\frac{101}{3520} x^{3}+10 k^{3}\right) a^{3}\right) \\
& -\frac{x^{9}}{45360}\left(\left(10 k^{2}+\frac{29}{440} x^{2}+\frac{13}{10} x k\right) a^{2}\right. \\
& \left.+\left(5 k+\frac{13}{40} x\right) a\right)
\end{aligned}
$$




$$
\begin{aligned}
& y_{5}=\frac{1}{1247400}\left(1+\left(\frac{589}{17472} x^{3} k^{3}+\frac{37}{208} x^{2} k^{4}\right.\right. \\
& \left.+\left(-\frac{1053}{112} x k+21 k^{2}-\frac{3257}{3360} x^{2}\right) a^{4}\right) x^{13}, \\
& +\frac{419}{26880} x^{4} k^{2}+\frac{19}{24} x k^{5} \\
& \left.+\frac{3431}{19009536} x^{6}+k^{6}+\frac{3431}{1118208} k x^{5}\right) a^{6} \\
& +\left(\frac{37}{52} x^{2} k^{3}+6 k^{5}+\frac{589}{5824} x^{3} k^{2}+\frac{3431}{1118208} x^{5}\right. \\
& \left.\left.+\frac{95}{24} x k^{4}+\frac{419}{13440} x^{4} k\right) a^{5}\right) x^{11} \\
& +\frac{1}{1247400}\left(\left(\frac{111}{104} x^{2} k^{2}+15 k^{4}+\frac{419}{26880} x^{4}\right.\right. \\
& \left.+\frac{589}{5824} x^{3} k+\frac{95}{12} x k^{3}\right) a^{4} \\
& +\left(20 k^{3}+\frac{95}{12} x k^{2}+\frac{37}{52} x^{2} k+\frac{589}{17472} x^{3}\right) a^{3} \\
& +\left(\frac{95}{24} x k+\frac{37}{208} x^{2}+15 k^{2}\right) a^{2} \\
& \left.+\left(\frac{19}{24} x+6 k\right) a\right) x^{11}, \\
& y_{6}=\frac{1}{97297200}\left(1+\left(-\frac{599}{60928} x^{4} k^{3}-\frac{351}{224} x k^{6}-\frac{1693}{26880} x^{3} k^{4}\right.\right. \\
& -\frac{3257}{3360} x^{2} k^{5}+k^{7}-\frac{35137}{2437120} x^{5} k^{2} \\
& \left.-\frac{124291}{46305280} x^{6} x-\frac{124291}{926105600} x^{7}\right) a^{7} \\
& +\left(-\frac{3257}{672} x^{2} k^{4}-\frac{1693}{6720} x^{3} k^{3}-\frac{35137}{1218560} x^{5} k\right. \\
& -\frac{1053}{112} x k^{5}-\frac{124291}{46305280} x^{6} \\
& \left.\left.+7 k^{6}-\frac{1797}{60928} x^{4} k^{2}\right)\right) x^{13} \\
& +\frac{1}{97297200} \\
& \times\left(a^{6}+\left(-\frac{3257}{336} x^{2} k^{3}+21 k^{5}-\frac{1693}{4480} x^{3} k^{2}\right.\right. \\
& \left.-\frac{35137}{2437120} x^{5}-\frac{1797}{60928} x^{4} k-\frac{5265}{224} x k^{4}\right) a^{5} \\
& +\left(-\frac{3257}{336} x^{2} k^{2}+35 k^{4}-\frac{599}{60928} x^{4}\right. \\
& \left.\left.-\frac{1693}{6720} x^{3} k-\frac{1755}{56} x k^{3}\right) a^{4}\right) x^{13} \\
& +\frac{1}{97297200} \\
& \times\left(\left(35 k^{3}-\frac{5265}{224} x k^{2}-\frac{3257}{672} x^{2} k-\frac{1693}{26880} x^{3}\right) a^{3}\right. \\
& y_{7}=-\frac{23}{9081072000} \\
& \times\left(1+\left(k^{8}-\frac{1875011}{808351272960} x^{8}+\frac{395}{552} x k^{7}\right.\right. \\
& -\frac{1875011}{35145707520} x^{7} k-\frac{392303}{4792596480} x^{6} k^{2} \\
& +\frac{270805}{68465664} x^{5} k^{3}+\frac{1037917}{51349248} x^{4} k^{4} \\
& \left.\left.+\frac{5075}{1351296} x^{3} k^{5}+\frac{8393}{225216} x^{2} k^{6}\right)\right) a^{8} x^{15} \\
& -\frac{23}{9081072000} \\
& \times\left(\frac{1037917}{808351272960} x^{4} k^{3}+\frac{8393}{37536} x^{2} k^{5}+8 k^{7}\right. \\
& +\frac{2765}{552} x k^{6}+\frac{25375}{1351296} x^{3} k^{4}-\frac{1875011}{35145707520} x^{7} \\
& \left.+\frac{270805}{22821888} x^{5} k^{2}-\frac{392303}{2396298240} x^{6} k\right) a^{7} x^{15} \\
& -\frac{23}{9081072000} \\
& \times\left(\frac{25375}{675648} x^{3} k^{3}+\frac{1037917}{8558208} x^{4} k^{2}\right. \\
& +\frac{270805}{22821888} x^{5} k+\frac{2765}{184} x k^{5} \\
& \left.+\frac{41965}{75072} x^{2} k^{4}-\frac{392303}{4792596480} x^{6}+28 k^{6}\right) a^{6} x^{15} \\
& -\frac{23}{9081072000} \\
& \times\left(\frac{41965}{56304} x^{2} k^{3}+56 k^{5}+\frac{25375}{675648} x^{3} k^{2}\right. \\
& +\frac{270805}{68465664} x^{5}+\frac{13825}{552} x k^{4} \\
& \left.+\frac{1037917}{12837312} x^{4} k+\frac{41965}{75072 a} x^{2} k^{2}\right) a^{5} x^{15} \\
& -\frac{23}{9081072000} \\
& \times\left(70 k^{4}+\frac{1037917}{51349248} x^{4}+\frac{25375}{1351296} x^{3} k\right. \\
& +\frac{13825}{552} x k^{3}+\frac{56 k^{3}}{a}+\frac{2765 x k^{2}}{184 a} \\
& \left.+\frac{8393 x^{2} k}{37536 a}+\frac{5075 x^{3}}{1351296 a}+\frac{2765 x k}{552 a^{2}}\right) a^{4} x^{15} \\
& -\frac{23}{9081072000}\left(\frac{8393}{225216} x^{6}+28 k^{2}\right) a^{2} x^{15}
\end{aligned}
$$




$$
-\frac{23}{9081072000}\left(\frac{395 x}{552}+8 k\right) a x^{15}
$$

and so on.

To exemplify, we will consider the seventh order approximation by substituting solutions (32) into (30) and calculating the limit when $p \rightarrow 1$ :

$$
y=\lim _{p \rightarrow 1}\left(\sum_{i=0}^{7} y_{i} p^{i}\right) .
$$

The above approximation is valid for all values of $k \geq$ 0 and corresponds to the results reported in [5] for the cases when $k=0$ and $k=20$. Clearly, if higher order approximations are considered, better accuracy is obtained but the resulting expressions could be too long.

5.2. HPM Laplace-Padé Scheme (LPHPM). Next, we study the case $k=0$; it means that $y^{\prime}(0)=1$ (see (26)). This is an interesting case because the following exact solution was reported in [59]:

$$
y(x)=1-\exp (-x)
$$

Thus, substituting $k=0$ in (33) we obtain the following series solution:

$$
\begin{aligned}
& y(x)=-0.5 x^{2}+x-7.878129877 \times 10^{-11} x^{19} \\
& +1.13972148927 \times 10^{-11} x^{20} \\
& +1.57691357363 \times 10^{-10} x^{18} \\
& +2.07320134446 \times 10^{-13} x^{21} \\
& -1.35120811977 \times 10^{-13} x^{22} \\
& +5.87481791204 \times 10^{-15} x^{23} \\
& +0.16666667 x^{3}-0.041666666 x^{4} \\
& +0.00833333333 x^{5}-0.00138888888 x^{6} \\
& +0.000198412698 x^{7}-0.0000248015873 x^{8} \\
& -2.755731 \times 10^{-7} x^{10}+0.000002755 x^{9} \\
& +2.5052108 \times 10^{-8} x^{11} \\
& -2.087675 \times 10^{-9} x^{12}+1.605904 \times 10^{-10} x^{13} \\
& -1.147074559 \times 10^{-11} x^{14} \\
& +7.647163731 \times 10^{-13} x^{15} \\
& -4.779477332 \times 10^{-14} x^{16} \\
& -5.073836906 \times 10^{-11} x^{17} \text {. }
\end{aligned}
$$

As we will see (35) is accurate only for small values of $x$. To guarantee the validity of the approximate solution (35) for large values of this variable, the series solution is transformed using the Padé approximation and Laplace transform (see Section 3). As first step, Laplace transform [54] is applied to (35):

$$
\begin{aligned}
\frac{-1}{s^{3}} & +\frac{1}{s^{2}}-\frac{9.583359 \times 10^{6}}{s^{20}}+\frac{2.77283070001 \times 10^{7}}{s^{21}} \\
& +\frac{1.009599 \times 10^{6}}{s^{19}}+\frac{1.0592181 \times 10^{7}}{s^{22}} \\
& -\frac{1.51875891 \times 10^{8}}{s^{23}}+\frac{1.51875891 \times 10^{8}}{s^{24}}+\frac{1}{s^{4}} \\
& -\frac{1}{s^{5}}+\frac{1}{s^{6}}-\frac{1}{s^{7}}+\frac{1}{s^{8}}-\frac{1}{s^{9}}-\frac{1}{s^{11}}+\frac{1}{s^{10}} \\
& +\frac{0.9999999998}{s^{12}}-\frac{1}{s^{13}}+\frac{0.9999999999}{s^{14}} \\
& -\frac{0.9999999997}{s^{15}}+\frac{1}{s^{16}}-\frac{1}{s^{17}}-\frac{18047}{s^{18}}
\end{aligned}
$$

and then, $s$ is substituted by $1 / x$ in the equation to obtain

$$
\begin{aligned}
& -x^{3}+x^{2}-9.583359 \times 10^{6} x^{20} \\
& +2.77283070001 \times 10^{7} x^{21}+1.009599 \times 10^{6} x^{19} \\
& +1.0592181 \times 10^{7} x^{22}-1.51875891 \times 10^{8} x^{23} \\
& +x^{4}-x^{5}+x^{6}-x^{7}+x^{8}-x^{9}-x^{11}+x^{10} \\
& +0.9999999998 x^{12}-x^{13}+0.9999999999 x^{14} \\
& -0.9999999997 x^{15}+x^{16}-x^{17}-18047 x^{18} \\
& +1.51875891 \times 10^{8} x^{24}
\end{aligned}
$$

Following Laplace-Padé scheme, Padé approximant [6/6] is applied to obtain

$$
\frac{x^{2}}{1+x}
$$

here $x$ is substituted by $1 / s$; the result is

$$
\frac{1}{s(s+1)} \text {. }
$$

Finally, by means of the inverse Laplace transform applied to (39), we obtain the exact solution (34) for (24).

5.3. HPM Padé Scheme (PHPM). Another way to recover lost information from the truncated series (33) is by means of applying the Padé approximant [7/7]. For the particular 
case when $k=0$ (see (35)), we obtain the following rational approximation for (24) (using dummy variables $a 1$ and $b 1$ )

$$
\begin{aligned}
a 1= & 1.15625115625 \times 10^{-7} x^{7} \\
& +0.000174825174825 x^{5} \\
& +0.0320512820513 x^{3}+x, \\
b 1= & 1+0.1153846153 x^{2}+0.001456876456 x^{4} \\
& +0.5 x+0.01602564102 x^{3} \\
& +0.00008741258741 x^{5} \\
& +0.00000323750323 x^{6} \\
& +5.781255781 \times 10^{-8} x^{7},
\end{aligned}
$$

and therefore, the final result is

$$
y(x)=\frac{a 1}{b 1} .
$$

\section{Discussion}

Figures 3 and 5 show a comparison between Runge Kutta 4 (RK4) numerical solution for different values of $k$ and approximations given by Laplace-Padé and Padé methods applied to (33). From Figures 4 and 6 it can be noticed that the relative error obtained by our approximations was low. As a matter of fact, the largest error for LPHPM is -0.006 when $k=5$ and for PHPM is -0.0250 when $k=0$. In a broad sense, if higher order approximations are considered, higher accuracy is obtained from LP-HPM and PHPM methods. In particular, it is noteworthy that Laplace-Padé method allows recovering the exact solution (34) when $k=0$ from the truncated series (33). This contrasts with Figure 2, which shows the comparison between RK4 and HPM approximations given by (33) for $k=0,0.3,1,2,5$ and $k=20$. It is evident that HPM series is accurate only for a restricted domain of values for the independent variable $x$. Also it is worth comparing the cumbersome approximation (35) for $k=0$, with the handy expressions (34) and (41) obtained by LHPM and PHPM, respectively. In [5] HPM was employed to solve (24) with a good approximation for a restricted domain of values for $x$ and small values for $k$, while in [6] the same equation was solved using perturbation method (PM) for small values of $k$. Indeed it is known that PM provides, in general, better results for small values of the perturbation parameter. Unlike the LPHPM and PHPM schemes were employed to obtain accurate solutions for different values of $k$, having low relative errors for $x$ values within the range $0 \leq x \leq 30$, as shown in Figures 4 and 6 .

The importance of providing analytical solutions, although approximate, with good accuracy is that numerical solutions only provide a qualitative idea of the problem to be solved. Besides, just as it was shown in one of our case studies, a solution obtained by a numerical method like Runge Kutta could hide the case of an exact solution. This

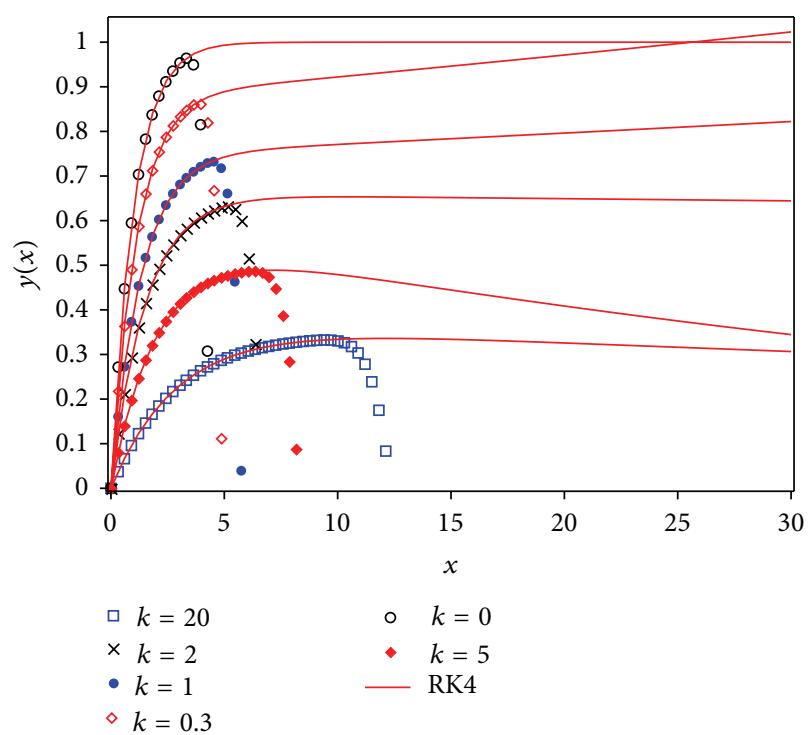

FIgURE 2: Fourth order Runge Kutta numerical solution for (24) (solid line) and HPM solution (33) (symbols).

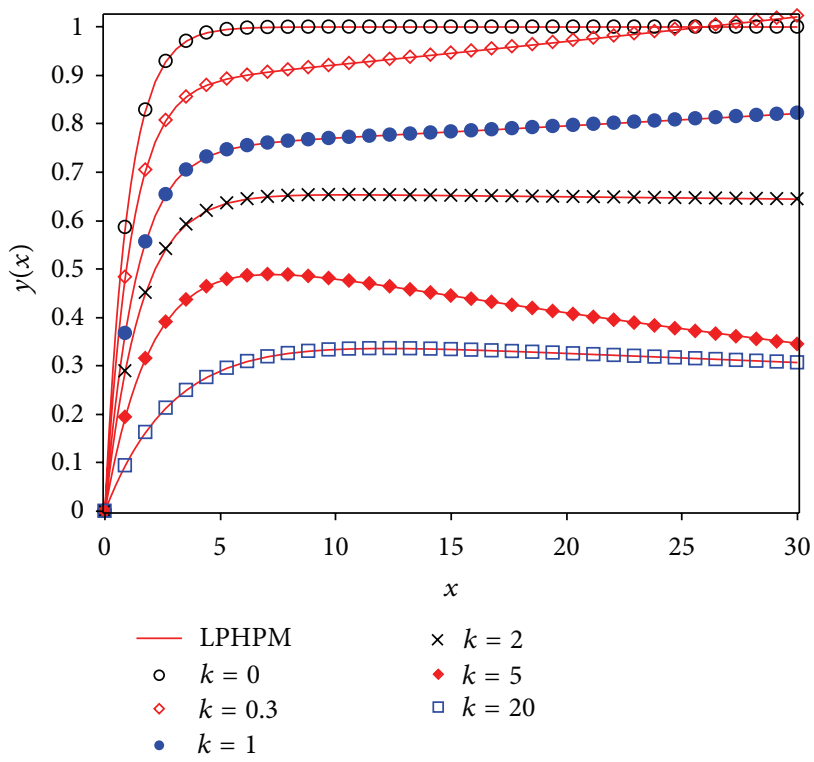

FIGURE 3: Fourth order Runge Kutta solution for (24) (symbols) and LPHPM (solid line).

possibility was successfully explored by LPHPM for case study $k=0$. Finally, another reason why we are interested in obtaining analytical approximation solutions is that nume rical algorithms could give some problems, such as numerical instabilities and oscillations, among others. This means that the numerical solutions may not correspond to the real solution of the original differential equation [60].

Just as it was seen with our HPM solutions, one disadvantage of approximating them with polynomials is its tendency to oscillate; this gives rise to the fact that the obtained solu tions diverge, especially for the case of problems defined on open intervals. This can be attributed to the possibility that 


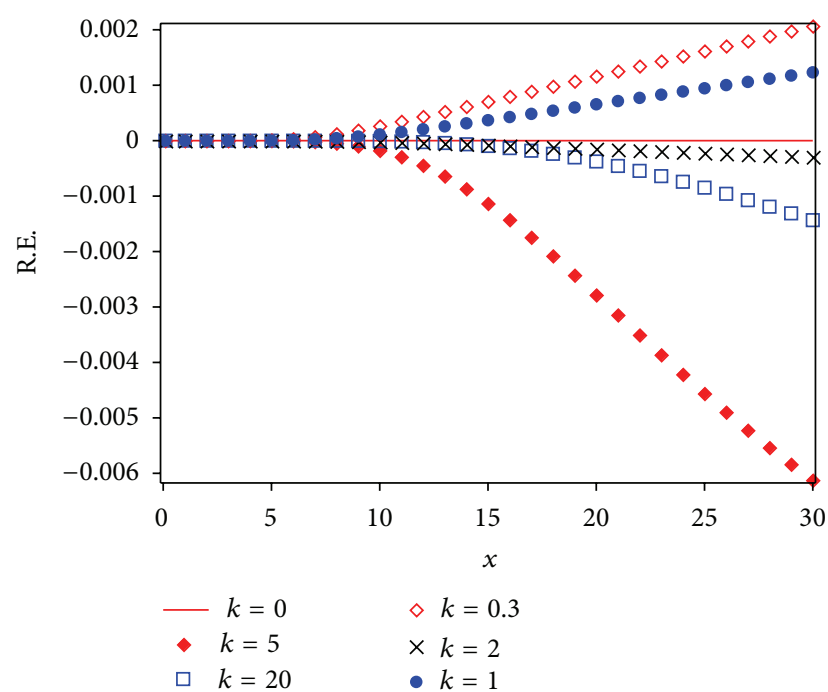

FIGURE 4: Relative error for different cases of LPHPM.

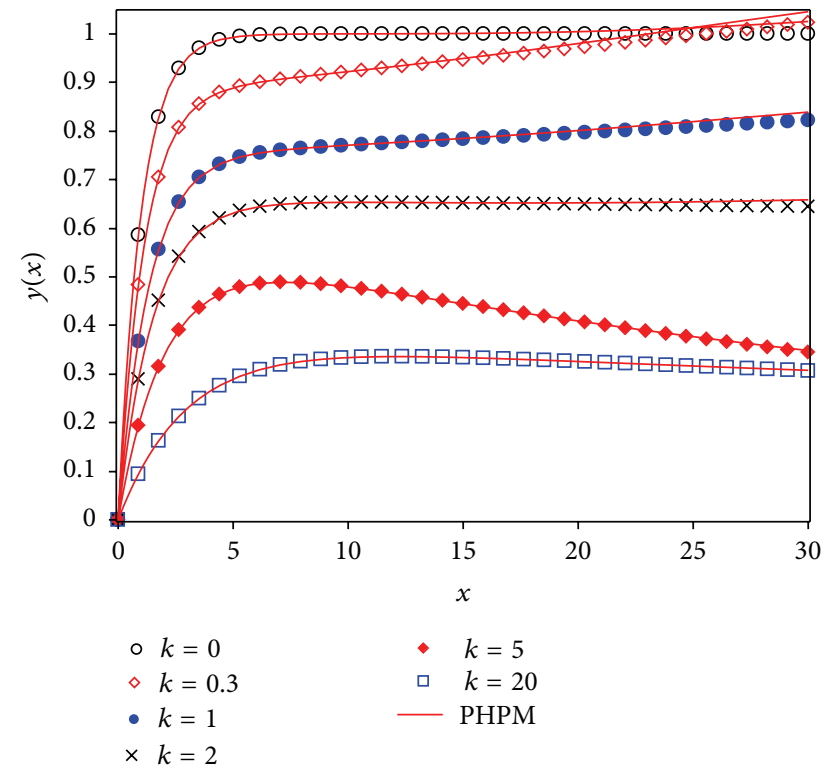

FIgURE 5: Fourth order Runge Kutta numerical solution for (24) (symbols) and PHPM (solid line).

the radius of convergence may not be sufficiently large to contain the boundaries of the domain of study $[56,61]$.

In order to improve the aforementioned, the use of rational functions was proposed, through the Padé approximation method.

The Padé approximation is an extension of the Taylor polynomial approximation but for rational functions. When the denominator of the approximation is a zero degree poly nomial function, the Padé approximation is reduced to a Maclaurin polynomial.

Padé-approximate extrapolation technique consists in approximating a truncated series (such as those resulting of HPM) by a rational function; the latter extends the range of validity of the initial polynomial. The above results are

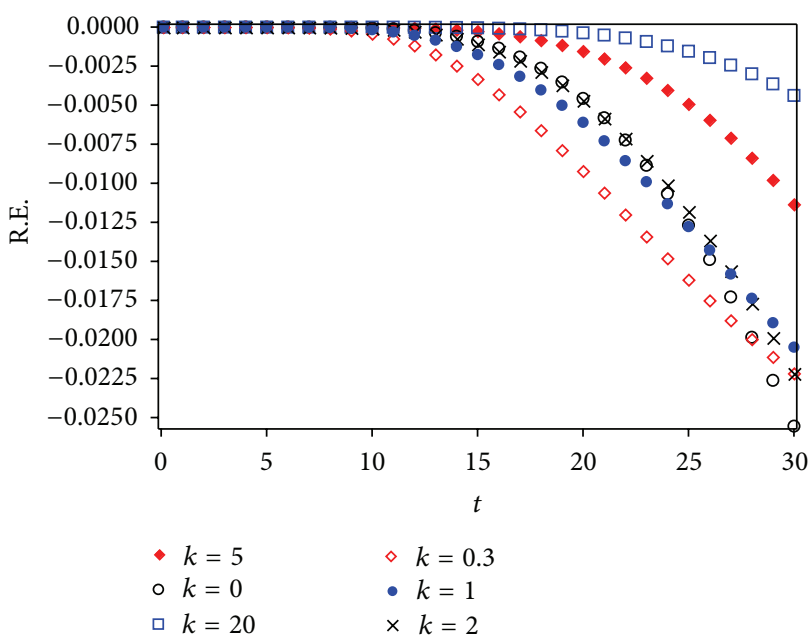

FIgURE 6: Relative error for different cases of PHPM.

particularly relevant if the truncated series represent the solution of a differential equation.

We observe that even though the series has a finite region of convergence, Padé approximant allows obtaining the limit of the function under study as $x$ takes large values, if $L=M$ (see Figures 3 and 5) [55]. In fact, the rational functions whose denominator and numerator have the same values of $L$ and $M$, or a degree almost identical, give rise to results better than those obtained by methods based on polynomial functions [61].

Finally, the convergence is uniform in any compact region in the case of Padé approximation, whereas truncated Maclaurin series is valid only in a near neighbourhood of zero point. The above explains why HPM-Padé has larger intervals of convergence in comparison with HPM series [55].

As it was already mentioned, LPHPM applies Padé approximant to the resulting expression, derived of applying Laplace transform to the HPM truncated series, and the rational function obtained in this way acquires the benefits of the abovementioned Padé method.

Nevertheless, one possible advantage of LPHPM is that the application of inverse Laplace transform at the last step of the method could result in the exact solution of the problem.

\section{Conclusions}

This work showed that some nonlinear problems may be adequately approximated using the coupling of the HPM method with Laplace-Padé and Padé methods to deal with HPM truncated power series. For instance, the flow induced by a stretching sheet is adequately described by our approximations given by LPHPM and PHPM (see Figure 3 through Figure 6). Figures 4 and 6 illustrate the relative error and show that the proposed solutions are highly accurate. Since that this procedure is, in principle, applicable to other similar problems, we conclude that LPHPM and PHPM are methods with high potential in the search for analytical approximate solutions for nonlinear problems. 


\section{Conflict of Interests}

The authors declare that there is no conflict of interests regarding the publication of this paper.

\section{Acknowledgment}

The authors gratefully acknowledge the financial support from the National Council for Science and Technology of Mexico (CONACyT) through Grant CB-2010-01 no. 157024.

\section{References}

[1] W. F. Hughes and J. A. Brighton, Dinámica De Los Fluidos, McGraw-Hill, New York, NY, USA, 1967.

[2] R. Resnick and D. Halliday, Física, vol. 1, John Wiley \& Sons, 1977.

[3] L. D. Landau and E. M. Lifschitz, Fluid Mechanics, vol. 6, Pergamon Press, 2nd edition, 1987.

[4] V. Aliakbar, A. Alizadeh-Pahlavan, and K. Sadeghy, "The influence of thermal radiation on MHD flow of Maxwellian fluids above stretching sheets," Communications in Nonlinear Science and Numerical Simulation, vol. 14, no. 3, pp. 779-794, 2009.

[5] M. M. Rashidi and D. D. Gangi, "Homotopy perturbation method for solving flow in the extrusion processes," IJE Transactions A: Basics, vol. 23, pp. 267-272, 2010.

[6] C. Y. Wang, "Flow due to a stretching boundary with partial slip-an exact solution of the Navier-Stokes equations," Chemical Engineering Science, vol. 57, no. 17, pp. 3745-3747, 2002.

[7] U. Filobello-Nino, H. Vazquez-Leal, Y. Khan et al., "A handy exact solution for flow due to a stretching boundary with partial slip," Revista Mexicana de Física E, vol. 59, no. 1, pp. 51-55, 2013.

[8] J. H. He, "A coupling method of a homotopy technique and a perturbation technique for non-linear problems," International Journal of Non-Linear Mechanics, vol. 35, no. 1, pp. 37-43, 2000.

[9] J.-H. He, "Homotopy perturbation technique," Computer Methods in Applied Mechanics and Engineering, vol. 178, no. 3-4, pp. 257-262, 1999.

[10] J.H. He, "Homotopy perturbation method for solving boundary value problems," Physics Letters A, vol. 350, no. 1-2, pp. 87-88, 2006.

[11] J.-H. He, "Recent development of the homotopy perturbation method," Topological Methods in Nonlinear Analysis, vol. 31, no. 2, pp. 205-209, 2008.

[12] A. Belendez, C. Pascual, M. L. Alvarez, D. I. Méndez, M. S. Yebra, and A. Hernández, "Higher order analytical approximate solutions to the nonlinear pendulum by He's homotopy method," Physica Scripta, vol. 79, no. 1, Article ID 015009, 24 pages, 2009.

[13] J.-H. He, "A coupling method of a homotopy technique and a perturbation technique for non-linear problems," International Journal of Non-Linear Mechanics, vol. 35, no. 1, pp. 37-43, 2000.

[14] M. El-Shahed, "Application of He's homotopy perturbation method to Volterra's integro-differential equation," International Journal of Nonlinear Sciences and Numerical Simulation, vol. 6, no. 2, pp. 163-168, 2005.

[15] J.-H. He, "Some asymptotic methods for strongly nonlinear equations," International Journal of Modern Physics B, vol. 20, no. 10, pp. 1141-1199, 2006.
[16] D. D. Ganji, H. Babazadeh, F. Noori, M. M. Pirouz, and M. Janipour, "An application of homotopy perturbation method for non linear blasius equation to boundary layer flow over a flat plate," International Journal of Nonlinear Science, vol. 7, pp. 309404, 2009.

[17] D. D. Ganji, H. Mirgolbabaei, M. Miansari, and M. Miansari, "Application of homotopy perturbation method to solve linear and non-linear systems of ordinary differential equations and differential equation of order three," Journal of Applied Sciences, vol. 8, no. 7, pp. 1256-1261, 2008.

[18] A. Fereidoon, Y. Rostamiyan, M. Akbarzade, and D. D. Ganji, "Application of He's homotopy perturbation method to nonlinear shock damper dynamics," Archive of Applied Mechanics, vol. 80, no. 6, pp. 641-649, 2010.

[19] P. R. Sharma and G. Methi, "Applications of homotopy perturbation method to partial differential equations," Asian Journal of Mathematics \& Statistics, vol. 4, no. 3, pp. 140-150, 2011.

[20] H. Aminikhah, "Analytical approximation to the solution of nonlinear Blasius' viscous flow equation by LTNHPM,' ISRN Mathematical Analysis, vol. 2012, Article ID 957473, 10 pages, 2012.

[21] H. Vázquez-Leal, U. Filobello-Niño, R. Castañeda-Sheissa, L. Hernández-Martínez, and A. Sarmiento-Reyes, "Modified HPMs inspired by homotopy continuation methods," Mathematical Problems in Engineering, vol. 2012, Article ID 309123, 19 pages, 2012.

[22] H. Vazquez-Leal, R. Castaneda-Sheissa, U. Filobello-Nino, A. Sarmiento-Reyes, and J. Sanchez Orea, "High accurate simple approximation of normal distribution integral," Mathematical Problems in Engineering, vol. 2012, Article ID 124029, 22 pages, 2012.

[23] U. Filobello-Nino, H. Vazquez-Leal, R. Castaneda-Sheissa et al., "An approximate solution of Blasius equation by using HPM method," Asian Journal of Mathematics and Statistics, vol. 2012, 10 pages, 2012.

[24] J. Biazar and H. Aminikhah, "Study of convergence of homotopy perturbation method for systems of partial differential equations," Computers \& Mathematics with Applications, vol. 58, no. 11-12, pp. 2221-2230, 2009.

[25] J. Biazar and H. Ghazvini, "Convergence of the homotopy perturbation method for partial differential equations," Nonlinear Analysis: Real World Applications, vol. 10, no. 5, pp. 2633-2640, 2009.

[26] U. Filobello-Nino, H. Vazquez-Leal, Y. Khan et al., "HPM applied to solve nonlinear circuits: a study case," Applied Mathematical Sciences, vol. 6, no. 87, pp. 4331-4344, 2012.

[27] M. A. Noor and S. T. Mohyud-Din, "Homotopy perturbation method for solving Thomas-Fermi equation using Pade approximants," International Journal of Nonlinear Science, vol. 8, no. 1, pp. 27-31, 2009.

[28] X. Feng, L. Mei, and G. He, "An efficient algorithm for solving Troesch's problem," Applied Mathematics and Computation, vol. 189, no. 1, pp. 500-507, 2007.

[29] S. H. Mirmoradi, I. Hosseinpour, S. Ghanbarpour, and A. Barari, "Application of an approximate analytical method to nonlinear Troesch's problem," Applied Mathematical Sciences, vol. 3, no. 32, pp. 1579-1585, 2009.

[30] M. Madani, M. Fathizadeh, Y. Khan, and A. Yildirim, "On the coupling of the homotopy perturbation method and Laplace transformation," Mathematical and Computer Modelling, vol. 53, no. 9-10, pp. 1937-1945, 2011. 
[31] M. Fathizadeh, M. Madani, Y. Khan, N. Faraz, A. Yildirim, and S. Tutkun, "An effective modification of the homotopy perturbation method for MHD viscous flow over a stretching sheet," Journal of King Saud University-Science, vol. 25, no. 2, pp. 107113, 2013.

[32] Y. Khan, Q. Wu, N. Faraz, and A. Yildirim, "The effects of variable viscosity and thermal conductivity on a thin film flow over a shrinking/stretching sheet," Computers \& Mathematics with Applications, vol. 61, no. 11, pp. 3391-3399, 2011.

[33] N. Faraz and Y. Khan, "Analytical solution of electrically conducted rotating flow of a second grade fluid over a shrinking surface," Ain Shams Engineering Journal, vol. 2, no. 3-4, pp. 221226, 2011.

[34] Y. Khan, H. Vazquez-Leal, and Q. Wu, "An efficient iterated method for mathematical biology model," Neural Computing and Applications, vol. 23, no. 3-4, pp. 677-682, 2013.

[35] S. Thiagarajan, A. Meena, S. Anitha, and L. Rajendran, "Analytical expression of the steady-state catalytic current of mediated bioelectrocatalysis and the application of He's homotopy perturbation method," Journal of Mathematical Chemistry, vol. 49, no. 8, pp. 1727-1740, 2011.

[36] L. M. B. Assas, "Approximate solutions for the generalized KdVBurgers' equation by He's variational iteration method," Physica Scripta, vol. 76, no. 2, pp. 161-164, 2007.

[37] J.-H. He, "Variational approach for nonlinear oscillators," Chaos, Solitons \& Fractals, vol. 34, no. 5, pp. 1430-1439, 2007.

[38] M. Kazemnia, S. A. Zahedi, M. Vaezi, and N. Tolou, "Assessment of modified variational iteration method in BVPs high-order differential equations," Journal of Applied Sciences, vol. 8, no. 22, pp. 4192-4197, 2008.

[39] R. Noorzad, A. T. Poor, and M. Omidvar, "Variational iteration method and homotopy-perturbation method for solving Burgers equation in fluid dynamics," Journal of Applied Sciences, vol. 8, no. 2, pp. 369-373, 2008.

[40] D. J. Evans and K. R. Raslan, "The tanh function method for solving some important non-linear partial differential equations," International Journal of Computer Mathematics, vol. 82, no. 7, pp. 897-905, 2005.

[41] F. Xu, "A generalized soliton solution of the KonopelchenkoDubrovsky equation using He's exp-function method," Zeitschrift fur Naturforschung A Journal of Physical Sciences, vol. 62, no. 12, pp. 685-688, 2007.

[42] J. Mahmoudi, N. Tolou, I. Khatami, A. Barari, and D. D. Ganji, "Explicit solution of nonlinear ZK-BBM wave equation using Exp-function method," Journal of Applied Sciences, vol. 8, no. 2, pp. 358-363, 2008.

[43] G. Adomian, "A review of the decomposition method in applied mathematics," Journal of Mathematical Analysis and Applications, vol. 135, no. 2, pp. 501-544, 1988.

[44] E. Babolian and J. Biazar, "On the order of convergence of Adomian method," Applied Mathematics and Computation, vol. 130, no. 2-3, pp. 383-387, 2002.

[45] A. Kooch and M. Abadyan, "Efficiency of modified Adomian decomposition for simulating the instability of nano-electromechanical switches: comparison with the conventional decomposition method," Trends in Applied Sciences Research, vol. 7, no. 1, pp. 57-67, 2012.

[46] A. Koochi and M. Abadyan, "Evaluating the ability of modified adomian decomposition method to simulate the instability of freestanding carbon nanotube: comparison with conventional decomposition method," Journal of Applied Sciences, vol. 11, no. 19, pp. 3421-3428, 2011.
[47] S. K. Vanani, S. Heidari, and M. Avaji, "A low-cost numerical algorithm for the solution of nonlinear delay boundary integral equations," Journal of Applied Sciences, vol. 11, no. 20, pp. 35043509, 2011.

[48] S. H. Chowdhury, "A comparison between the modified homotopy perturbation method and adomian decomposition method for solving nonlinear heat transfer equations," Journal of Applied Sciences, vol. 11, no. 7, pp. 1416-1420, 2011.

[49] Y. C. Jiao, Y. Yamamoto, C. Dang, and Y. Hao, "An aftertreatment technique for improving the accuracy of Adomian's decomposition method," Computers \& Mathematics with Applications, vol. 43, no. 6-7, pp. 783-798, 2002.

[50] L.-N. Zhang and L. Xu, "Determination of the limit cycle by He's parameter expansion for oscillators in a potential," Zeitschrift für Naturforschung-Section A Journal of Physical Sciences, vol. 62, no. 7, pp. 396-398, 2007.

[51] T. Patel, M. N. Mehta, and V. H. Pradhan, “The numerical solution of Burger's equation arising into the irradiation of tumour tissue in biological diffusing system by Homotopy Analysis Method," Asian Journal of Applied Sciences, vol. 5, no. 1, pp. 60-66, 2012.

[52] H. N. Hassan and M. A. El-Tawil, "An efficient analytic approach for solving two-point nonlinear boundary value problems by homotopy analysis method," Mathematical Methods in the Applied Sciences, vol. 34, no. 8, pp. 977-989, 2011.

[53] U. Filobello-Nino, H. Vazquez-Leal, Y. Khan et al., "Using perturbation methods and Laplace-Padé approximation to solve nonlinear problems," Miskolc Mathematical Notesc, vol. 14, no. 1, pp. 89-101, 2013.

[54] B. Benhammouda, H. Vazquez-Leal, L. Benhamouche et al., "Procedure for exact solutions of stiff ordinary differential equations systems," The British Journal of Mathematics \& Computer Science, vol. 4, no. 23, pp. 3252-3270, 2014.

[55] A. Kelleci and A. Yıldırım, "An efficient numerical method for solving coupled Burgers' equation by combining homotopy perturbation and Pade techniques," Numerical Methods for Partial Differential Equations, vol. 27, no. 4, pp. 982-995, 2011.

[56] B. Raftari, H. Adibi, and A. Yildirim, "Solution of the MHD Falkner-Skan flow by Adomian decomposition method and Padé approximants," International Journal of Numerical Methods for Heat and Fluid Flow, vol. 22, no. 8, pp. 1010-1020, 2012.

[57] H. Vazquez-Leal, B. Benhammouda, U. Filobello-Nino et al., "Direct application of Padé approximant for solving nonlinear differential equations," SpringerPlus, vol. 3, article 563, 2014.

[58] J. Vleggaar, "Laminar boundary-layer behaviour on continuous, accelerating surfaces," Chemical Engineering Science, vol. 32, no. 12, pp. 1517-1525, 1977.

[59] L. J. Crane, "Flow past a stretching plate," Zeitschrift für Angewandte Mathematik und Physik, vol. 21, no. 4, pp. 645-647, 1970.

[60] H. Vazquez-Leal and F. Guerrero, "Application of series method with Padé and Laplace-Padé resummation methods to solve a model for the evolution of smoking habit in Spain," Computational \& Applied Mathematics, vol. 33, no. 1, pp. 181-192, 2014.

[61] J. Faires and R. Burden, Numerical Methods, Brooks/Cole, Boston, Mass, USA, 4th edition, 2012. 


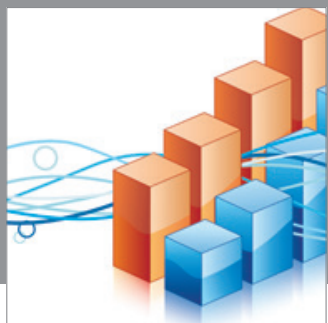

Advances in

Operations Research

mansans

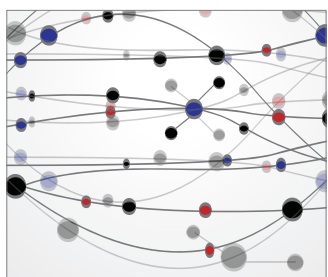

The Scientific World Journal
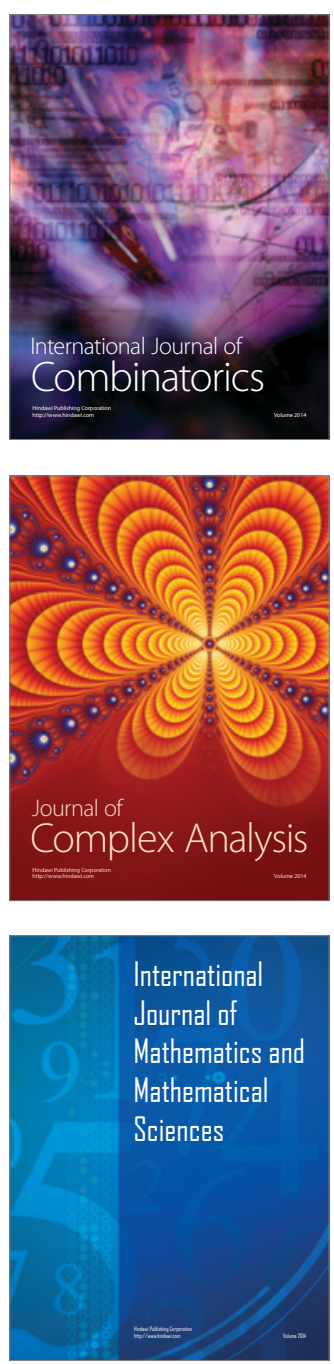
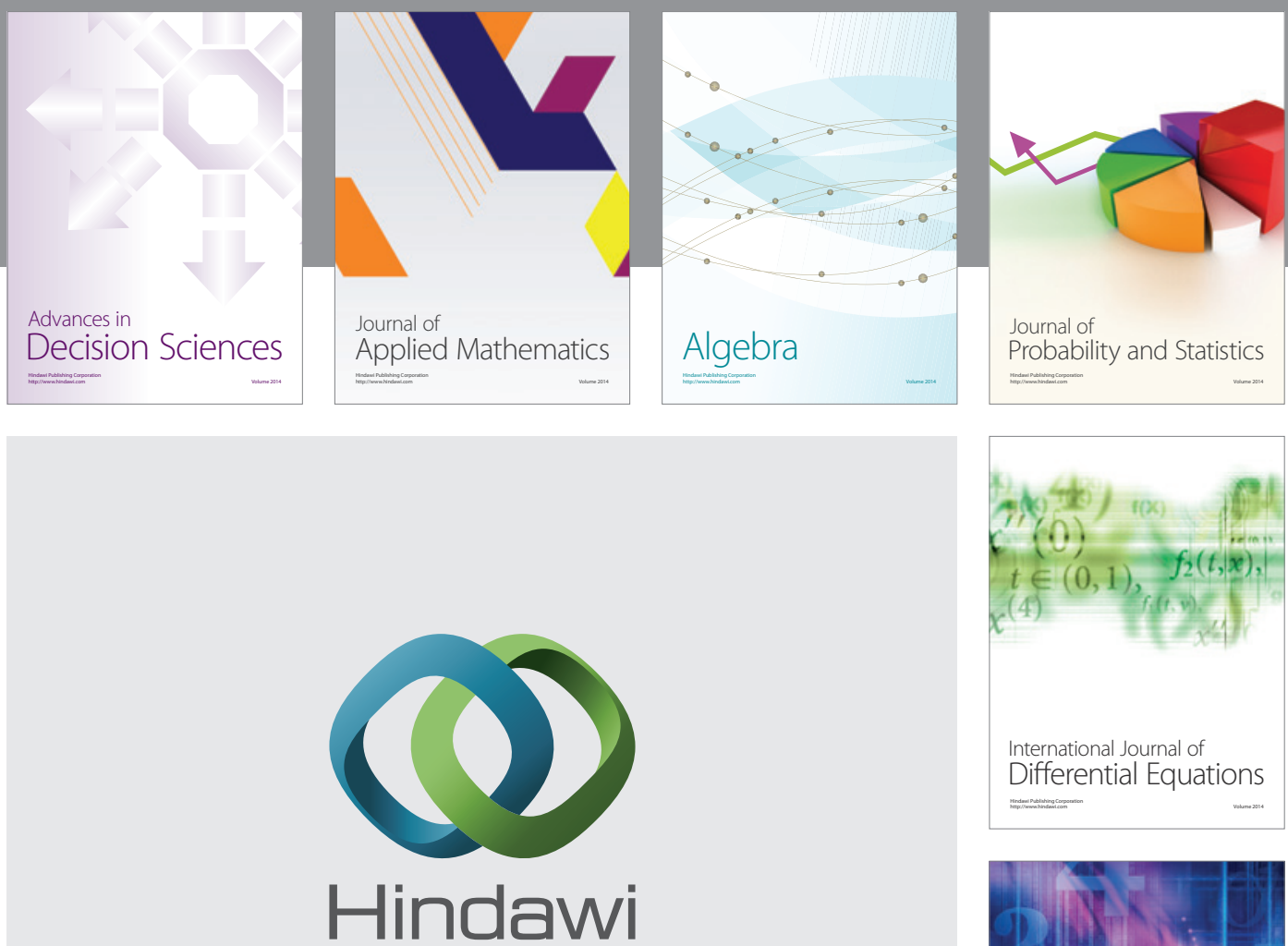

Submit your manuscripts at http://www.hindawi.com
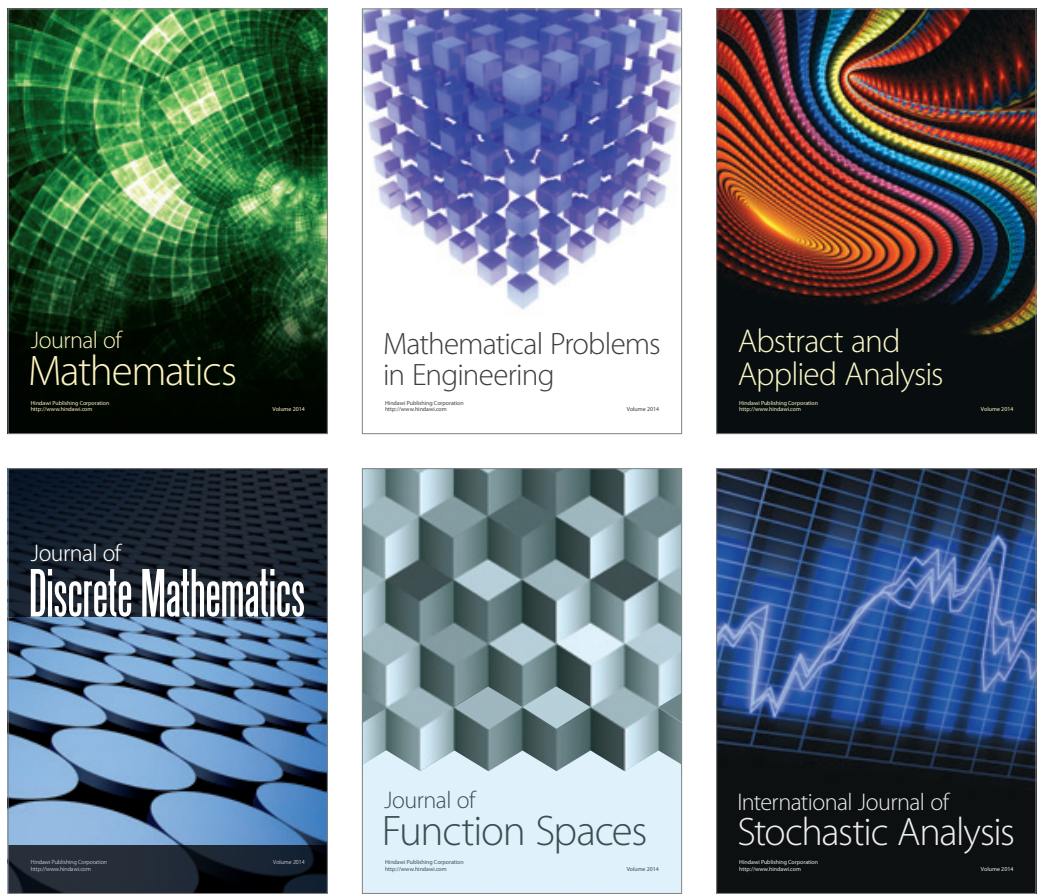

Journal of

Function Spaces

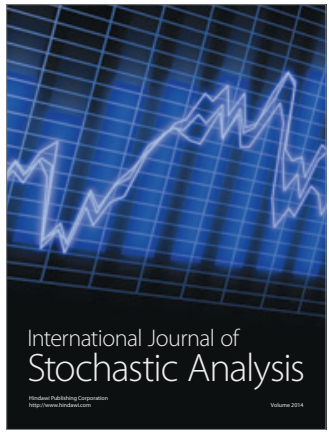

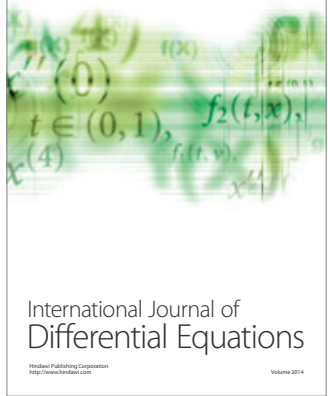
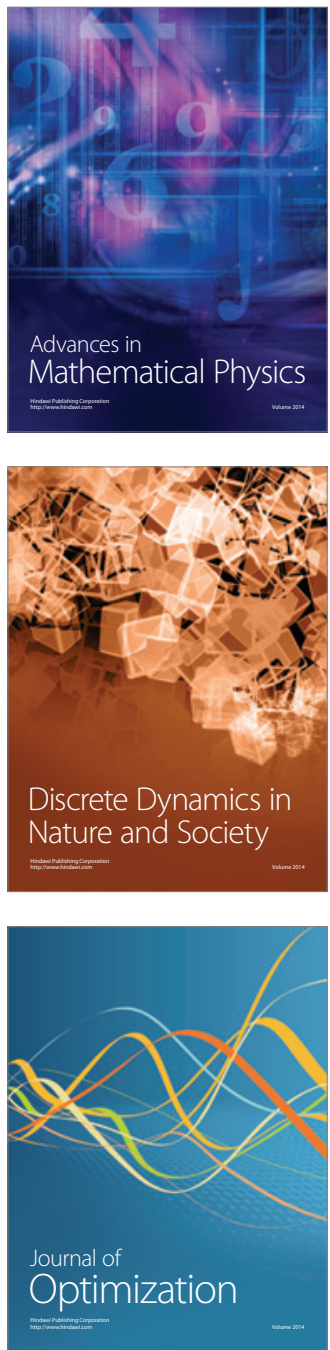